\title{
具有三轨输出的三值触发器及其 在三值时序电路中的应用
}

\author{
吴训威陈华雄 \\ (杭州大学物理系)
}

掩要

本文指出三值触发器 (tri-flop) 应具有三轨输出、三态预置能力、合理的逻辑功 能与激励表等. 以此要求为前提, 本文提出了与二值触发器对应的各种三值触发器 的设计,其中包括三值基本触发器(Latch) 及各种类型的三值主从触发器, 如置态型、 $D$ 型、 $J K L$ 型与 $T$ 型等。这些触发器的结构及逻辑功能与以前提出的各种触发器的 设计都不同，在本文中还通过实例表明，由于它们具有合理的激励表，因此能方便地 应用于各种三值时序电路的设计。

\section{一、引 言}

近年来对多值逻辑的研究已引起重视, 并已成为近代数字电子学的一个研究方向．这是 因为多值逻辑能提供一个丰富的逻辑结构，从而为应用于物理系统提供了可观的潜能 ${ }^{[1,2 l}$. 从 工程的观点看, 多值数字系统的最重要的优点是增加单线携带信息的能力,从而起到减少 LSI 及数字系统的引线数的作用.

在对多值逻辑最初的研究中所遇到的电路实现上的困难，随着大规模集成电路技术的发 展，正在逐渐得到解决.然而，多值逻辑迄今为止仍然只在有限的场合下使用，并不能充分显示 出期望中的作用. 分析其原因,认为对于多值逻辑付诸实用的主要障碍之一就是缺乏合适的、 且不复杂的存储单元 ${ }^{31}$. 在有关多值逻辑的论著中, 仅有少数才涉及存储单元与时序电路方面 的讨论. 为此, 作者认为, 作为对基数更高的多值逻辑进行研究的第一步, 对三值触发器及其 在三值时序电路中的应用进行研究似是一个较有意义的研究课题, 其中特别是对于三值触发 器的研究,乃是更重要的基础研究。

从二值触发器的设计与研究中吸取启发似已成为大部分三值触发器的研究方法．如果综 合以往的研究工作 ${ }^{[4-8]}$,则似可把主要的研究结果归纳成如下几点:

1. 所研究的触发器类型有基本触发器 (Latch 或直接耦合 $R S$ 触发器)，D型 Latch 及脉冲 模式工作的主从触发器, 其中包括 $R S$ 触发器、 $D$ 型触发器、 $J K$ 触发器及 $T$ 型触发器等.

2. 大多数多值触发器都是由多值门电路构成的，其中基本存储单元是由二个多值反相门 
（与非门或者或非门）通过交叉耦合而成.

3. 大多数触发器具有二个互补输出, 即 $Q$ 与 $\bar{Q}$. 主从兔发器的输入除了激励输入外, 尚 有时钟输入 $C L$, 而 $C L$ 通常是一个二值信号.

4. 各种类型的触发器根据其特定结构具有跟相应的二值触发器相似的各种逻辑功能与激 领表,利用这些激励表就可以进行三值时序电路的设计.

但是,在使用已提出的各种触发器设计时序电路时仍遇到了困难。这些困难包括:

（1）各种设计的主从触发器的预置功能不理想. Mouftah 等人 ${ }^{[8]}$ 提出了如图 1 所示结构 的 $P Z N$ 三值基本触发器 (tri-Latch). 图中或非门符号内的数字 3 表示为三值或非门. 该图暗 示了对触发器直接置态的输人方式. 显然, 用 $Z=1$ 进行置 1 是不理想的, 这一方面是由于在 时序电路中不能通过一个单一的预置按钮和单一的预置输入 2 预置初态; 另一方面，如果考虑 到该触发器还须接受其他输入信号, 输入 $Z=1$ 就可能起不到置 1 作用.

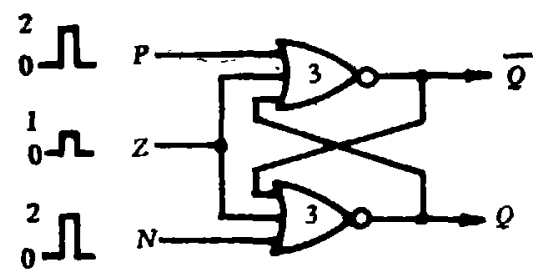

图 $1 \quad P Z N$ 三值基本触发器

（2）触发器的激励表不理想. 这一点主要表现在具有较强逻辑功能的多激励输入触发器 ( $R S$ 触发器与 $J K$ 触发器) 的使用上. Acha 等已提出了多值 $J K$ 触发器与 $R S$ 触发器的激励 表 ${ }^{[5,7]}$, 但在讨论中发现, 为了实现某一特定的状态转换,激励输人变量可能有多种相互约束的 取法,并且出现了激励变量可以具有部分任意取值的情况. 很清楚, 这会在使用这些触发器设 计时序电路时带来不便.

（3）某些触发器的“互补”输出不符合多值数字系统的需要. 如果多值触发器用于构成诸 如计数器等时序电路, 则它们的输出一般用作组合电路的输入. 对基于与一或一阈运算的代 数系统的组合电路来说，输人变量以 ${ }^{i} x^{i}$ 的多轨形式更符合需要; 对基于模代数 ${ }^{[9]}$ 的组合电路 来说, 输人变量以变量各种极的多轨形式更符合需要 ${ }^{[10]}$. 因此, 以往提出的多值触发器的“互 补”输出并不符合多值数字系统的需要. 顺便指出, 对二.值触发器而言, 通常的互补输出 $(Q$, $\bar{Q})$ 正好就是状态变量的二个阈运算 $\left({ }^{1} Q^{1},{ }^{0} Q^{0}\right)$, 并且也正好是状态变量的二个极性表示 ${ }^{[11]}$.

因此, 我们认为现有的三值触发器设计较难满足三值时序电路的设计需要, 故新型的三值 触发器应该满足如下基本要求:

1. 它应该具有完善的预置功能, 可通过单一的预置电平来预置触发器到任何一个状态 $(0$, $1,2)$.

2. 它应有合理的逻辑功能，从而具有理想的敨励表，以利于三值时序电路的设计.

3. 它应有合理的多轨输出, 以符合实现相应于给定代数系统的组合电路的输入需要.

此外, 期待中的三值触发器的结构与逻辑功能还应具有可与二值触发器相对应的特征。综 上所速，我们确定将各种三值触发器的新设计及发展相应的三值时序电路设计程序作为本文 研究的宗旨. 


\section{二、三值逻辑的代数系统}

设定在三值逻辑中, 常数、变量、函数 $\in\{0,1,2\}$. 基本的单变量运算为:

$$
\begin{gathered}
\text { 阈运算 }{ }^{i} x^{i}= \begin{cases}0 & (x \neq i), \\
2 & (x=i),\end{cases} \\
i \in\{0,1,2\} . \\
\text { 反相运算 } \vec{x}=2-x .
\end{gathered}
$$

基本的多变量运算为:

与运算

$$
\begin{aligned}
& x \cap y=\min (x, y), . \\
& x \cup y=\max (x, y) . \\
& x \oplus y=(x+y)_{\bmod , 3 .} \\
& x \cdot y=(x \times y)_{\bmod , 3 .}
\end{aligned}
$$

模 3 加运算

模 3 乘运算

前面诸式中的一,,,$\times$ 分别为算术减、算术加与算术乘.

三值逻辑中的常用代数系统为：（a）以模 3 乘和模 3 加为基本运算的代数系统；(b)以与j 一或一阈运算为基本运算的代数系统. 其中后者与传统的与一或一非二值系统相对应，且较 为实用与重要. 在我们的讨论中,将选用后一多值代数系统.

在该代数系统中的诸基本定理、关系式及它们的证明已为众所周知,因此，在此仅着重提 出三个与间运算有关的定理予以讨论.

定理 1. $\left({ }^{0} x^{0} \cap{ }^{1} x^{1}\right)=\left({ }^{0} x^{0} \cap{ }^{2} x^{2}\right)=\left({ }^{1} x^{1} \cap{ }^{2} x^{2}\right)=0$,

$$
{ }^{0} x^{0} \cup^{1} x^{1} \cup^{2} x^{2}=2
$$

定理 2. ${ }^{i} x^{\prime}=i \oplus \prime(x \oplus i)^{i \oplus \prime}$,

$$
i, i \in\{0,1,2\} \text {. }
$$

定理 3. $f\left(x_{1}, x_{2} \cdots x_{n}\right)=\left[f\left(0, x_{2}, \cdots x_{n}\right) \cap{ }^{0} x_{1}^{0}\right] \cup\left[f\left(1, x_{2} \cdots x_{n}\right) \cap{ }^{1} x_{1}^{1}\right] \cup[f(2$,

$$
\begin{aligned}
& \left.\left.x_{2} \cdots x_{n}\right) \cap^{2} x_{1}^{2}\right], \\
f\left(x_{1}, x_{2} \cdots x_{n}\right)= & {\left[f\left(0, x_{2} \cdots x_{n}\right) \cup{ }^{0} x_{1}^{0}\right] \cap\left[f\left(1, x_{2} \cdots x_{n}\right) \cup^{\overline{1} x_{1}^{1}}\right] } \\
& \cap\left[f\left(2, x_{2} \cdots x_{n}\right) \cup^{2} x_{1}^{2}\right] .
\end{aligned}
$$

定理 3 表示了任意函数可能通过对变量的逐次分解使具有最小项展开与最大项展开的形式, 此外,它还表示了间运算 ' $x^{i}$ 乃是实现组合函数的基本运算. 定理 2 是显然的. 而定理 1 则表 示了阈运算之间的互斥性, 即不论变量取何值,三个单变量运算 ${ }^{0} x^{0},{ }^{1} x^{1},{ }^{2} x^{2}$ 中只有一个取非零 值“2”。容易证明下式成立:

$$
{ }^{i} x^{2}=\overline{x^{i} \cup x^{k}} . \quad(i \neq j \neq k)
$$

上式表示三者中有一个不是独立的,事实上,确实只要用其中三个便能得到三值输人变量 $x$ :

$$
\dot{x}={ }^{2} x^{2} \cup\left(1 \cap^{1} x^{1}\right) \text {. }
$$

综上所述，我们可以把一个三值信号分解成三个二值信号 ${ }^{2} x^{2},{ }^{1} x^{1}$ 及 ${ }^{0} x^{0}$ 进行三轨二值传 送. 它作为组合电路的输人，可能为组合函数的综合提供方便. 这个情况完全类似于二值组 合电路中的双轨输人 $x, \bar{x}$, 即 ${ }^{1} x^{1},{ }^{0} x^{0}$ 。联系到上一节的讨论, 现已逐渐清楚, 如棵把二值触发器 中的互补输出 $Q, \bar{Q}$ 看作是实现二个國运算 ${ }^{1} Q^{1},{ }^{0} Q^{0}$ 的双轨输出, 则适合所选代数係统的三 值触发器的正确设计似应具有三轨输出 ${ }^{2} Q^{2},{ }^{1} Q^{1}$ 及 ${ }^{0} Q^{0}$. 


\section{三、具有三轨输出的三值基本触发器（Latch）}

考虑到阈运算 ' $x^{i}$ 的二值性, 我们可用由三个二值或非门交叉耦合的三稳态触发器 ${ }^{[2]}$ 来构 成三轨输出的基本触发器 ( Latch), 如图 2 所示. 在正常使用时,图中的三个置态输入 $s_{i} \in\{0$, $2\},(i=0,1,2)$, 且必须满足互斥关系,即约束条件:

$$
s_{i} \cdot S_{j}=0^{\mathrm{D}} . \quad(i \neq i)
$$

图 2 所示的三值 Latch 的逻辑功能是简明的, 当 $S_{i}=2$ 时三值 Latch 置数为 $Q_{+}=i$, 而当 $S_{0}=S_{1}=S_{2}=0$ 时, 三值 Latch 处于稳定的存储状态，即 $Q_{+}=Q$. 显然，象二值 I.atch一样， 如果置态输人违反约束条件 (12) 式, 则三值 Latch 就不能正常工作. 三值 Latch 逻辑功能的代

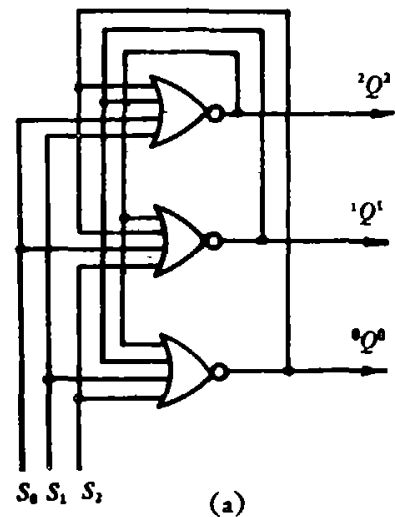

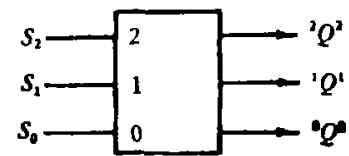

(b)

图 2 三轨输出的三值基本触发器

(a) 逻辑图, (b) 图形符号

数表示式为:

$$
Q_{+}=0 \cdot S_{0}+1 \cdot S_{1}+2 \cdot S_{2}+\bar{S}_{0} \cdot \bar{S}_{1} \cdot \bar{S}_{2} \cdot Q,
$$

由于 $S_{0}, S_{1}, S_{2} \in\{0,2\}$ ，上式便可化简为:

$$
\begin{aligned}
& Q_{+}=S_{2}+1 \cdot S_{1}+\bar{S}_{0} \cdot \bar{S}_{1} \cdot Q . \\
& \quad\left(S_{0} \cdot S_{1}=S_{0} \cdot S_{2}=S_{1} \cdot S_{2}=0\right)
\end{aligned}
$$

括号内的附加等式即为必须遵循的约束条件. 注意到二值 Latch 的逻辑功能表示为:

$$
Q_{+}=S+\bar{R} \cdot Q .(R \cdot S=0)
$$

显然,如果把式中的 $R, S$ 分别记成 $S_{0}$ 与 $S_{1}$, 则它们就有与 (13)式相对应的形式. (13)式还可 写成如下的分解形式:

$$
\left.\begin{array}{c}
{ }^{2} Q_{+}^{2}=S_{2}+\bar{S}_{0} \cdot \bar{S}_{1} \cdot \bar{S}_{2} \cdot{ }^{2} Q^{2}=S_{2}+\bar{S}_{0} \cdot \bar{S}_{1} \cdot{ }^{2} Q^{2}, \\
{ }^{1} Q_{+}^{1}=S_{1}+\bar{S}_{0} \cdot \bar{S}_{1} \cdot \bar{S}_{2} \cdot{ }^{1} Q^{1}=S_{1}+\bar{S}_{0} \cdot \bar{S}_{2} \cdot{ }^{1} Q^{1}, \\
{ }^{0} Q_{+}^{0}=S_{0}+\bar{S}_{0} \cdot \bar{S}_{1} \cdot \bar{S}_{2} \cdot{ }^{0} Q^{0}=S_{0}+\bar{S}_{1} \cdot \bar{S}_{2} \cdot{ }^{0} Q^{0}
\end{array}\right\}
$$

如果对图 2 所示的三轨三值 Latch 的三个输入通过二值与门用时钟脉冲予以控制, 则可得

1）为了表示的简明，从本节开始用,$+ \cdot$ 分别表示或运算及与运算. 
到如图 3(a)所示的钟控三值 Latch, 图中当 $C L=2$ 时由 $S_{0}, S_{1}, S_{2}$ 对三值 Latch 进行置数, 而 当 $C L=0$ 时三值 Latch 便处于稳定的存储状态.

如果在图 3(a)的输入与输出分别加接三值信号译码器与编码器, 便可构成如图 3(b) 所 示的钟控 $D$ 型 Latch，图中的虚线部分即为图 3(a) 所示的钟控三值 Latch. 钟控 $D$ 型 Latch 实 现了信号的单轨输人与输出,它的逻辑功能与二值 $D$ 型 Latch 有同样的表示,即

$$
Q_{+}=D \text {. }
$$

图 4 给出了三值信号译码器与编码器的 MOS 电路设计, 在二值情况下, 由于 ${ }^{4} x^{1}=x$ 及 ${ }^{0} x^{0}=\bar{x}$, 因此译码器内只含一只反相器, 而编码器则可取消.

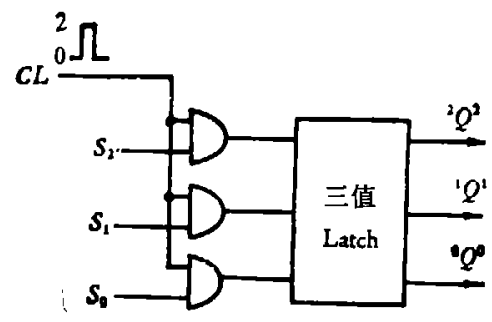

(a)

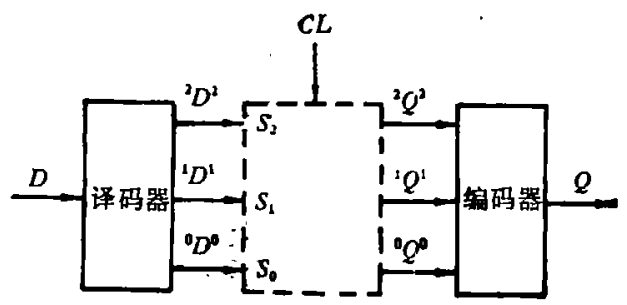

(b)

图 3 （a）钟控三值 Latch，(b) 钟控 $D$ 型 Latch

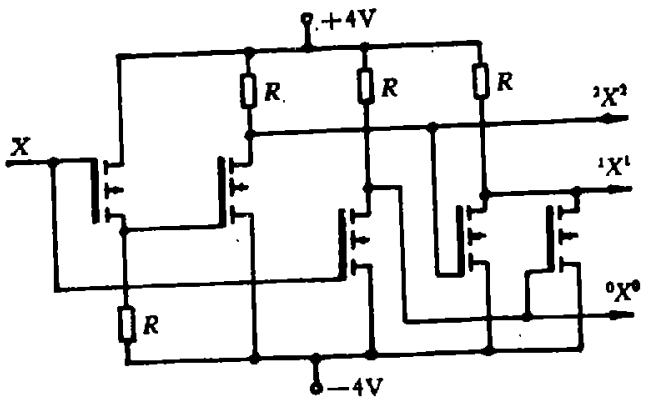

(a)

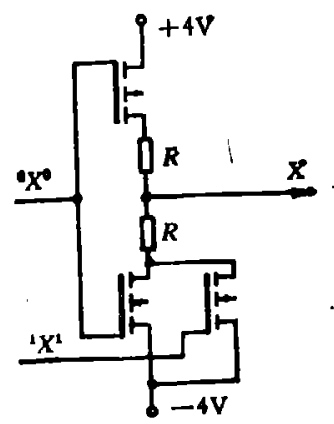

(b)

图 4 (a) MOS 三值信号译码器, (b) MOS 三值信号编码器

\section{四、三值主从触发器}

把二个由图 3(a) 所示的钟控三值 Latch 前后串接, 便可构成如图 5(a)所示的置态型主从 触发器. 它的工作原理是这样的: 当 $C L$ 从低电平变化到高电平时,主触发器的状态发生变 化, 而从触发器处于稳定的存储状态; 当 $C L$ 从高电平变化到低电平时, 情况则相反. 因此, 该触发器是在时钟脉冲 $C L$ 下跳时改变状态的.二个三值 Latch 的三个直接耦合输人 $S_{0 D}, S_{1 D}$ 和 $S_{2 D}$ 是用来预置初态的, 这只要增加图 2 中或非门的扇人便可达到。显然, 置态型主从触发 器对应于二值 $R S$ 主从触发器, 它的逻辑功能与对输入的约束条件可由(13)式予以表示. 


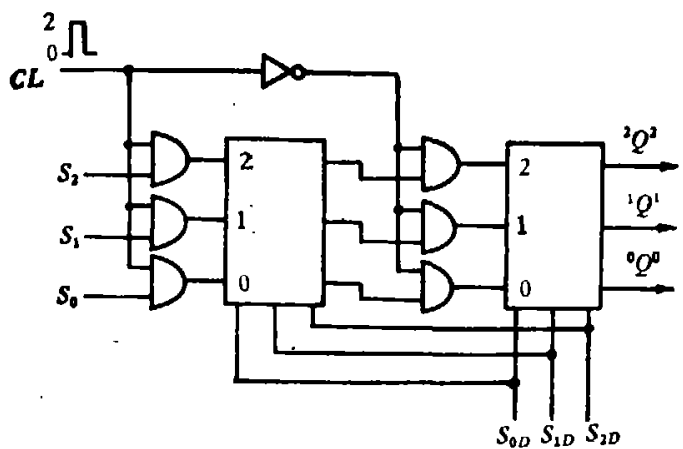

(a)

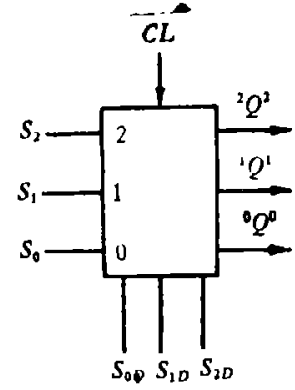

(b)

图 5 三值置态型主从触发器

(a) 逻辑图，（b）图形符号

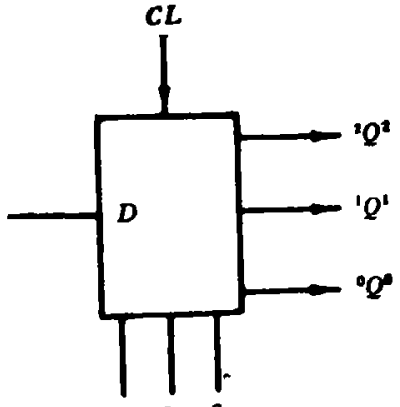

$S_{\mathrm{OD}} S_{10} S_{1 \mathrm{D}}$

图 6 三值 $D$ 型触发器 的图形符号

如果在图 5 所示的触发器输入端加接三值信号译码器, 便可构成三值 $D$ 型触发器. 它的 图形符号如图 6 所示,而它的逻辑功能可由(15)式予以表示.

根据前面的讨论, 现在已可猜测到与逻䐜功能最强的二值触发器- $J K$ 触发器对应的三 值触发器应该具有三个放励输入, 假设它们为 $J, K, L$, 且 $J, K, L \in\{0,1,2\}$. 只要在三值 置态型触发器的输入辅以适当的组合电路,并使三值触发器的三轨输出予以适当反馈, 便能实 现期待中的三值 $J K L$ 触发器. 现取

$$
\left.\begin{array}{l}
S_{2}={ }^{2} J^{2} \cdot{ }^{0} Q^{0}+{ }^{1} K^{1} \cdot{ }^{1} Q^{1}, \\
S_{1}={ }^{1} J^{1} \cdot{ }^{0} Q^{0}+{ }^{2} L^{2} \cdot{ }^{2} Q^{2}, \\
S_{0}={ }^{2} K^{2} \cdot{ }^{1} Q^{1}+{ }^{1} L^{1} \cdot{ }^{2} Q^{2} .
\end{array}\right\}
$$

由(7)式与(16)式不难证明, 不论 $J, K, L$ 取何值，均能使 $S_{0}, S_{1}, S_{2}$ 满足(14)式中的约束关系. 此外, 还可由(14)式得到:

$$
\left.\begin{array}{l}
{ }^{2} Q_{+}^{2}={ }^{2} J^{2} \cdot{ }^{0} Q^{0}+{ }^{1} K^{1} \cdot{ }^{1} Q^{1}+{ }^{0} L^{0} \cdot{ }^{2} Q^{2}={ }^{2} J^{2} \cdot{ }^{0} Q^{0}+{ }^{2}(K \oplus 1){ }^{2} \cdot{ }^{1} Q^{1}+{ }^{2}(L \oplus 2)^{2} \cdot{ }^{2} Q^{2}, \\
{ }^{1} Q_{+}^{1}={ }^{1} J^{1} \cdot{ }^{0} Q^{0}+{ }^{0} K^{0} \cdot{ }^{1} Q^{1}+{ }^{2} L^{2} \cdot{ }^{2} Q^{2}={ }^{1} J^{1} \cdot{ }^{0} Q^{0}+{ }^{1}(K \oplus 1)^{1} \cdot{ }^{1} Q^{1}+{ }^{1}(L \oplus 2)^{1} \cdot{ }^{2} Q^{2}, \\
{ }^{0} Q_{+}^{0}={ }^{0} J^{0} \cdot{ }^{0} Q^{0}+{ }^{2} K^{2} \cdot{ }^{1} Q^{1}+{ }^{1} L^{1} \cdot{ }^{2} Q^{2}={ }^{0} J^{0} \cdot{ }^{0} Q^{0}+{ }^{0}(K \oplus 1)^{0} \cdot{ }^{1} Q^{1}+{ }^{0}(L \oplus 2)^{0} \cdot{ }^{2} Q^{2} .
\end{array}\right\}
$$

在上式中的后一等号是利用 (8) 式得出的. 可把 (17) 式给出的分解形式合并成一个状态方 程:

$$
Q_{+}=J \cdot{ }^{0} Q^{0}+(K \oplus 1) \cdot{ }^{1} Q^{1}+(L \oplus 2) \cdot{ }^{2} Q^{2},
$$

上式表示了三值 $J K L$ 触发器的逻辑功能. 显然, 它与二值 $J K$ 触发器的次态方程

$$
Q_{+}=J \cdot \bar{Q}+\overline{\boldsymbol{K}} \cdot Q=J \cdot{ }^{0} Q^{0}+(K \oplus 1) \cdot{ }^{1} Q^{1}
$$

是完全对应的. 图7(a)表示了用图 5 所示的三值置态型触发器构成的三值 $J K L$ 触发器. 图中 用了三个三值信号译码器来获得 ${ }^{2} J^{2},{ }^{1} J^{1},{ }^{2} K^{2},{ }^{1} K^{1},{ }^{2} L^{2},{ }^{1} L^{1}$, 而触发器的其余部分则均由二值 门电路构成. 图 7(b) 表示了它的图形符号. 它与图 5(b) 的明显不同之处在于它的三个激倣 输入为三值信号, 而图 5(b) 中的三个激励输入为二值信号.

完全类似于二值触发器的情况, 把 $J, K, L$ 联结在一起便可构成三值 $T$ 型触发器. 在 (18) 式中代人 $J=K=L=T$ 便可得到三值 $T$ 型触发器的次态方程如下: 


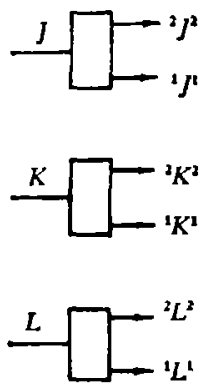

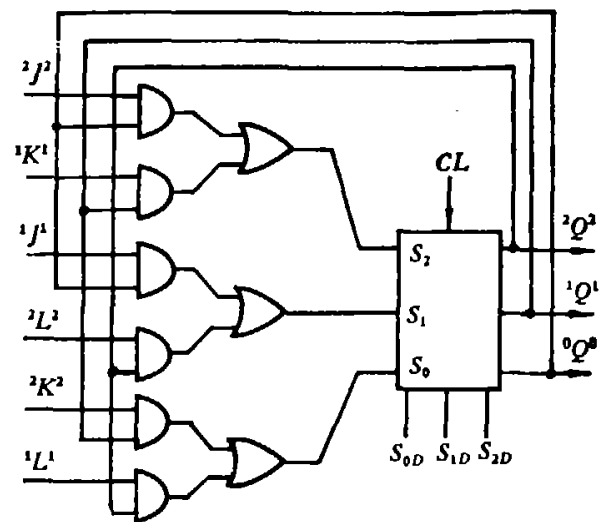

(a)

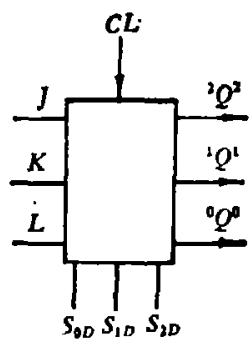

(b)

图 7 三值 $J K L$ 触发器

(a)逻辑图，（b）图形符号

$$
Q_{+}=T \oplus Q \text {. }
$$

显然, 当 $T=0$ 时触发器状态保持不变, $T=1$ 时触发器作加法计数, 而 $T=2$ 时触发器作减 法计数. 三值 $T$ 型触发器也能以类似于图 7(a) 的方式直接由三值置态型触发器构成. 但是, 由于 $J=K=L=T$, 因此只需用一个三值信号译码器。它也可直接用二个二值信号 ${ }^{2} T^{2}$ 及 ${ }^{1} T^{1}$ 作激励输入, 这时三值 $T$ 型触发器就全部由二值门电路构成. $T$ 型触发器的图形符号如 图 8 所示:

表 1 各种三值主从触发器的激励表

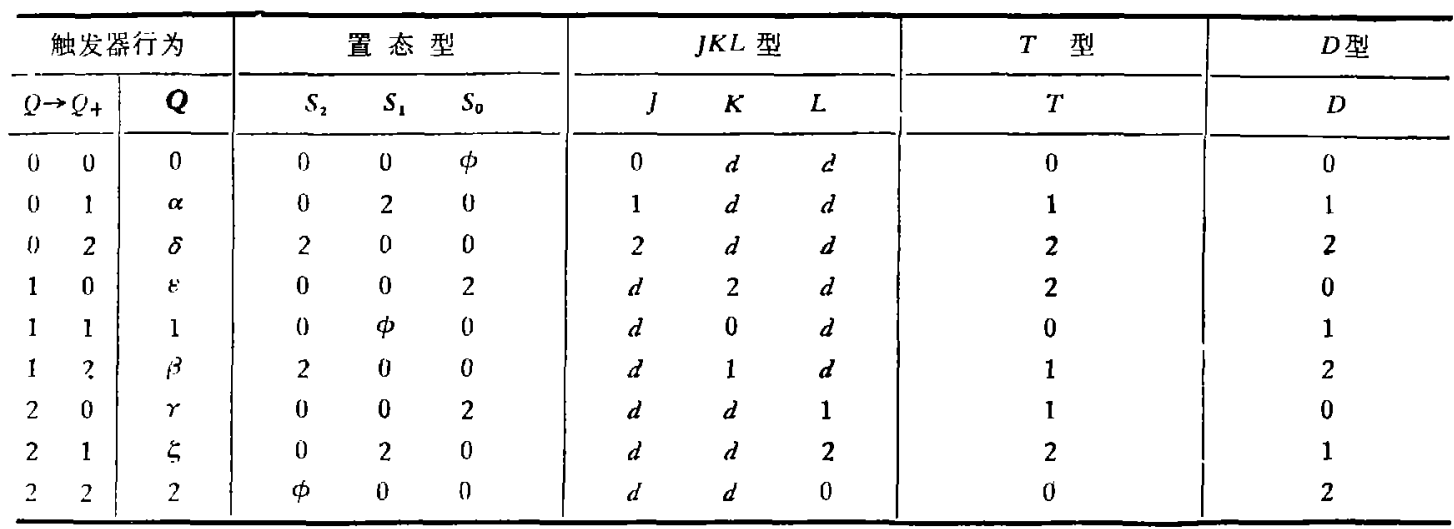

根据(13),(15),(18)与 (19) 诸式表示的各种三值触发器的逻辑功能,不难得到如表 1 所 示的各种三值主从触发器的激励表. 表中九种符号 $0,1,2, \alpha, \beta, \gamma, \delta, \varepsilon, \zeta$ 分别用来表示九种 特定的触发器行为,数字表示触发器状态保持不变,而希腊字母用来表示触发器特定的状态转 换。此外,还用 $\phi$ 与 $d$ 分别表示二值 $\{0,2\}$ 与三值 $\{0,1,2\}$ 情况下的任意取值条件. 表 1 表示 了前面提出的四种三值主从触发器不仅具有完善的预置功能及合理的三轨输出, 而且具有理 想的, 且可与二值触发器相对应的激励表, 所有这一些均为使用这些三值触发器设计三值时序 电路清除了障㥂。 


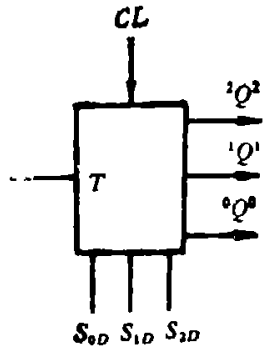

(a)

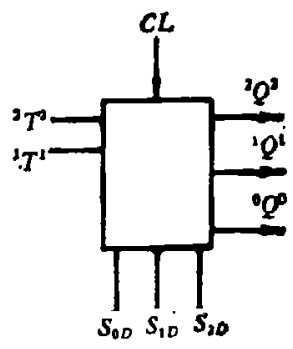

(b)

图 8 三值 $T$ 型触发器的图形符号

\section{五、三值时序电路的设计}

由于上节中提出的四种三值主从触发器与二值主从触发器具有理想的对应关系，因此在 二值时序电路中的一套设计方法均可方便地用于三值时序电路的设计. 下面将通过例子具体 说明三值时序电路的设计方法.

例 1 九进制加法计数器设计

表 2 九进制加法计数器的次态表与状态转换表

\begin{tabular}{|c|c|c|c|c|c|}
\hline$A$ & $B$ & $A_{t}$ & $B_{t}$ & $\boldsymbol{A}$ & $\boldsymbol{B}$ \\
\hline 0 & 0 & 0 & 1 & 0 & $\alpha$ \\
\hline 0 & 1 & 0 & 2 & 0 & $\beta$ \\
\hline 0 & 2 & 1 & 0 & $\alpha$ & $r$ \\
\hline 1 & 0 & 1 & 1 & 1 & $\alpha$ \\
\hline 1 & 1 & 1 & 2 & 1 & $\beta$ \\
\hline$\ldots 1$ & 2 & 2 & 0 & $\beta$ & $r$ \\
\hline 2 & 0 & 2 & $\mathbf{I}$ & 2 & $\alpha$ \\
\hline 2 & 1 & 2 & 2 & 2 & $\beta$ \\
\hline 2 & 2 & 0 & 0 & $r$ & $\gamma$ \\
\hline
\end{tabular}

由于 $3^{2}=9$, 因此只要用二只三值触发器 $A, B$ 便可实现具有九个状态的九进制加法计数 器。表 2 给出了次态表与状态转换表,而图 9(a),(b) 表示二个三值触发器的状态转换图. 如

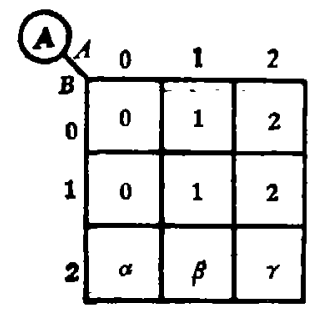

(a).

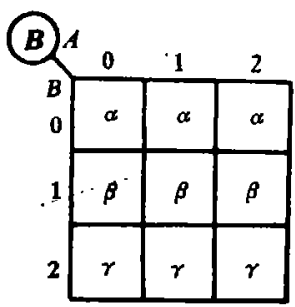

(b)

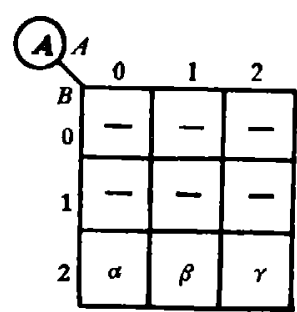

(c)

图 9 触发器的状态转换 $\mathrm{K}$ 图

(a), (b) $C L$ 为时钟, (c) $r_{B}$ 为时钟 
果使用三值 $\mathrm{T}$ 型触发器, 则根据表 1 所示的激励表不难得到每个触发器的激励函数:

$$
\left.\begin{array}{l}
T_{A}=1 \cdot{ }^{2} B^{2}, \\
T_{B}=1 .
\end{array}\right\}
$$

图 10(a) 表示了九进制同步加法计数器的逻辑图. 图中二个三值触发器 $A$ 与 $B$ 用同一时 钟脉冲 $C L$ 进行同步触发, 且在三值触发器 $A$ 的输入端用了一个三值与门. 此外, 清零按针 的作用使三值触发器 $A, B$ 能预置 $(0,0)$ 的初态.

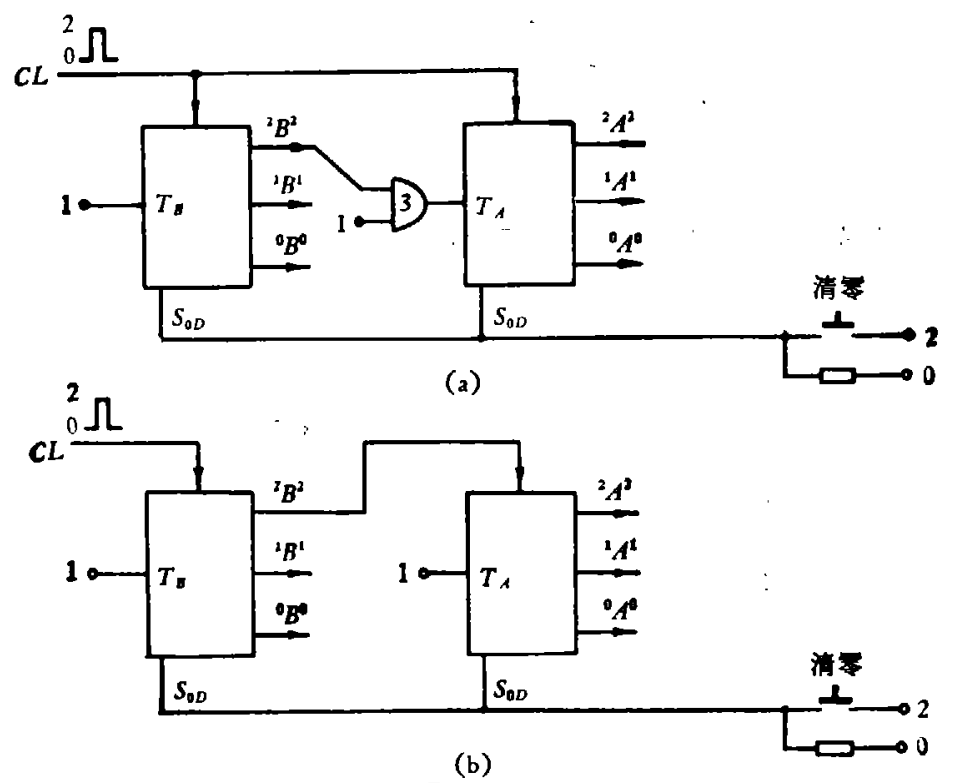

图 10 (a) 九进制同步加法计数器逻辑图 (b) 九进制异步加法计数器逻辑图

如果注意到在表 2 中三值触发器 $B$ 的 $\gamma_{B}$ 行为全覆盖三值触发器 $A$ 的所有翻转行为, 也 即 $\gamma_{B} \supseteq\left\{\alpha_{A}, \beta_{A}, \gamma_{A}\right\}$, 那末, 触发器 $B$ 的 $\gamma_{B}$ 行为显然可供作触发器 $A$ 的异步时钟, 并且可以 期望触发器 $A$ 将有较简单的激励函数. 在 $\gamma_{B}$ 以外的各个时钟周期内, 触发器 $A$ 由于没有触发 脉冲, 能不受激励输入的控制而保持原状态, 因此, 图 9(a) 可改画成图 9(c). 图中的短划表 示相应状态时激励函数允许自由取值. 由图 9(c)可得到: $T_{A}=1$. 图 10(b)表示厂异步九进 制加法计数器的逻辑图. 图中 ${ }^{2} B^{2}$ 用作触发器 $A$ 的时钟信号. 显然, 它与二值逻辑中的纹波式

表 3 八进制加法计数器的次态表与状态转换表

\begin{tabular}{|c|c|c|c|c|c|}
\hline$A$ & $B$ & $A_{t}$ & $B_{t}$ & $A$ & $B$ \\
\hline 0 & 0 & 0 & 1 & 0 & $\alpha$ \\
\hline 0 & 1 & 0 & 2 & 0 & $\beta$ \\
\hline 0 & 2 & 1 & 0 & $a$ & $\gamma$ \\
\hline 1 & 0 & 1 & 1 & 1 & $\alpha$ \\
\hline 1 & $\mathbf{I}$ & 1 & 2 & 1 & $\beta$ \\
\hline 1 & 2 & 2 & 0 & $\beta$ & $r$ \\
\hline 2 & 0 & 2 & 1 & 2 & $\alpha$ \\
\hline 2 & 1 & 0 & 0 & $r$ & $\varepsilon$ \\
\hline 2 & 2 & $x$ & $x$ & $x$ & $x$ \\
\hline
\end{tabular}


计数器相对应.

例 2 八进制加法计数器设计

由于 $3^{2}>8$, 因此也只需二只三值触发器 $A, B$ 便可实现八进制加法计数器. 表 3 给出了 次态表与状态转换表, 而图 11 表示了二个三值触发器 $A$ 与 $B$ 的状态转换图. 表与图中的记 号 $\times$ 表示资余态. 如果使用三值 $J K L$ 触发器, 则根据表 1 所示的激励表不难得到二个三值 触发器的激励函数:

$$
\left.\begin{array}{l}
J_{A}=K_{A}=1 \cdot{ }^{2} B^{2}, \\
L_{A}=1 \cdot{ }^{1} B^{1}, \\
J_{B}=L_{B}=1, \\
K_{B}=1+{ }^{2} A^{2} .
\end{array}\right\}
$$

应该指出, 如果最小化程序正确, 那末三值 $J K L$ 触发器的激励函数就不会含有本触发器

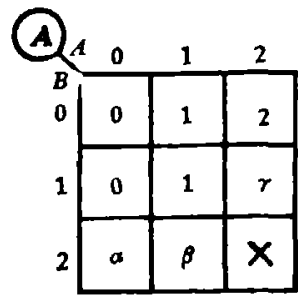

(a)

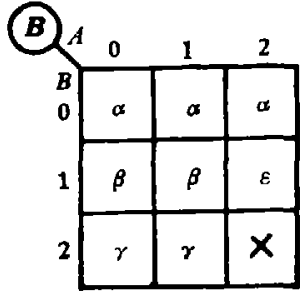

(b)

图 11 触发器的状态转换 $\mathrm{K}$ 图
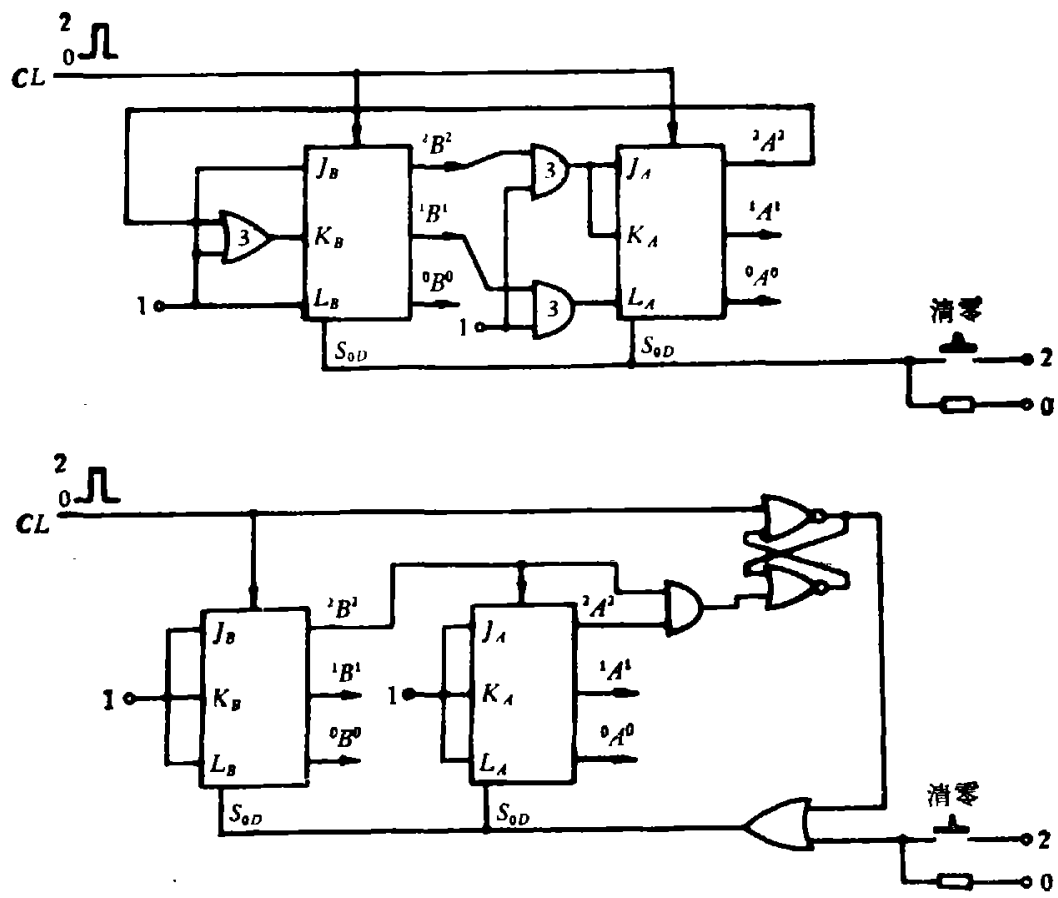

图 12 (a)八进制同步加法计数器的逻辑图 (b) 八进制反馈型加法计数器的逻辑图 
的输出, 如 (21) 式所示。这一点可从 (16) 式或表 1 得到证明,而该性质是与二值 $J K$ 触发器 一样的.

图 12(a) 表示八进制同步加法计数器的逻辑图，类似于二值计数器的情况，由于表 3 所 示的计数时序符合按三进制数的正常加法计数次序,因此,可在图 10(b) 所示的纹波式计数器 的基础上加以反馈复位来实现反馈型八进制加法计数器, 如图 12(b) 所示. 可以注意到图中 门电路均为二值的, 并且二个三值触发器也可以用三值 $\mathrm{T}$ 型触发器来代替.

\section{六、结 论}

三值触发器是三值时序电路的基本存储元件, 因此, 获得三值触发器的正确设计将对设计 三值时序电路具有根本重要的意义.

本文的讨论证明了, 在一开始就明确对三值触发器的要求是合理的。如果只是完全模仿 二值触发器的结构, 简单地用三值门电路代替二值门电路去设计具有互补输出的三值触发器, 则将如引言中指出的那样,由于它们既缺乏完善的预置能力, 又缺乏合理的激励表 (对三值 RS 触发器与三值 $J K$ 触发器), 从而会给三值时序电路的设计带来困难. 而本文根据预定设计要 求设计的三值触发器则能避免这些困难。

本文设计的所有三值触发器的基本部分都是由二值门电路构成的. 由此带来的好处是可 以利用已有的二值电路技术，另外一个附带的好处是由于二值 $\{0,2\}$ 的时钟信号 $C L$ 作用于二 值门,因此, $C L$ 在变化时瞬时出现的中间电平 1 不会象对三值门电路那样引起问题.

本文设计的各种三值触发器都具有二值的三轨输出, 这对三值组合电路的综合是有利的. 并且, 由于它们的二值特点才使之能如图 10(b) 所示可以直接作为另一个三值触发器的异:步 时钟, 而不需加接特殊的负三值反相器 $N T I^{[8]}$.

三值触发器在简单用作信号的存储与传输时,三轨输出或许是不必要的,此时可象图3(b) 那样使用一个三值信号编码器使改为单轨输出. 事实上, 当三值触发器用作信号传输, 如用 $D$ 型触发器构成移位寄存器时, 完全可以采用以前的三值触发器设计方案. 它们使用图 1 所示 的三值或非门交叉耦合结构为基本存储器, 并且只需使用一个输出信号 $Q$. 由于 $D$ 型触发器 的激励表没有困难,并且对移位寄存器来说往往只需要清零的预置功能,因此引言中分析的困 难就不会遇到.

与以前提出的各种设计相比, 本文设计的各种三值触发器与传统的二值触发器存在更密 切的对应关系，而正由于这一特点才使得此处讨论的三值时序电路的设计程序类同于二值情 况, 这一点已由前面的实例予以证明.

最后指出, 本文提出的对三值触发器的设计与应用完全可以推广到更高基数的情况.

本文在准备过程中得到英国 BATH 大学 S. L. Hurst 教授的鼓励与有益的指教, 作者谨 在此表示衰心感谢.

\section{参考文嗝}

[1] Rine, D. C., Computer Science and Multiple-Valued Logic Theory and Applications, Amsterdam, The Netherlands, North-Holland, 1977.

[2] Vtanesic, Z. G. and Smith, K. C., Computer, 7(1974), 34-41. 
[ 3 ] Vranesic, Z- G., IEEE Trans. Comput., C-26(1977), 1181-1182.

[ 4 ] Wojcik, A. S., Proc. 1974 Int. Symp. on Muitiple-Valucd Logic, 1974, $285-302$.

[ 5 ] Acha, J. I. and Huertas, J. L., Electron Lett., 11 (1975), 624.

[6] Irving, T: A., Sbiva, S. G. and Nagle, H. T., IEEE Trats. Comput, C-25(1976), $237-246$.

[ 7 ] Acha, J. I. and Huertas, J. L., ibid., C-25(1976), 759-763.

[ 8 ] Mọuftah, H. T. and Jordan, I. B., ibid., C-26(1977), 281-288.

[ 9 ] Hurst, S. L., Logical Processing of Digital Signals, Crane Russak, N. Y. and Edward Arnold, London, 1978.

[10]吴训威、陈华雄, 中国科学 A 辑, 1983, 9:847-856.

[11] Wu X., Chen, X. and Hurst, S. L., IEE Proc., Pt. E, 129(1982), 15-20.

[12] Richards, R. K., Digital Design, John Wiley and Sons, Inc., N. Y., 1971. 